Kurzbeiträge

Ernst-Bernd Blümle

\title{
Das Ressourcenportfolio der NPO
}

\author{
Versuch einer Systematik der Inputs
}

Beiträge; Finanzmittel; Finanzmittelsurrogate; Geldspenden; Ressourcenarten; Ressourcenmix; Sach- und Dienstleistungsspenden; Zeitspenden

Die Nonprofit-Organisationen, die zwischen Unternehmen/Haushalten und dem Staat angesiedelt sind, bilden einen heterogenen Sektor. Die Typenvielfalt ist komplex. Für diese Gebilde soll im Folgenden ein Raster der möglichen organisationsspezifischen Ressourcenarten vorgestellt werden, der für Analyse- und Planungszwecke genutzt werden kann.

\section{Einführung}

In zunehmendem Maße wird von der Politik, den Medien und der Öffentlichkeit, aber auch von der Wissenschaft die wirtschaftliche wie gesellschaftspolitische Bedeutung des Dritten Sektors erkannt. So ist beispielsweise die Caritas mit über 500.000 Beschäftigten der größte private Arbeitgeber in Deutschland.

Unbestritten ist: Der Dritte Sektor ist weltweit auf Wachstumskurs und hat seit Jahren überdurchschnittlich zum Beschäftigungswachstum beigetragen. So ist die Beschäftigung in den acht im Rahmen des Johns Hopkins Nonprofit Comparative Sector Projektes untersuchten Ländern (Belgien, Deutschland, Frankreich, Großbritannien, Israel, Japan, Niederlande und USA) zwischen 1990 und 1995 durchschnittlich um 23 Prozent oder über vier Prozent pro Jahr gestiegen. Im Vergleich dazu wuchs die übrige Wirtschaft in diesen Ländern nur um sechs Prozent oder kaum mehr als ein Prozent im Jahr.

Ziel dieses Beitrages ist es, einerseits eine Systematik des gesamten organisationsspezifischen Inputs vorzustellen, andererseits kann dieser Ansatz der Ressourcenplanung und Ressourcensuche dienen. 


\section{NPOs und ihre Ressourcen}

\section{Nonprofit-Organisationen}

Die vorherrschenden Rechtsformen dieser Organisationen, die sich zwischen Markt und Staat angesiedelt haben, sind der eingetragene und nicht-eingetragene Verein, die Genossenschaft und die Stiftung. Die Erscheinungsvielfalt wie die Ausprägungsformen sind ausgesprochen groß: Neben den mitgliederorientierten NPO (Verbände der Wirtschaftsund Arbeitswelt, Vereine des Freizeitbereiches) stehen die drittorientierten Organisationen. Die Träger sind nicht gleichzeitig Nutznießer der Aktivität (z.B. soziale Organisationen). Als Organisationen der gesellschaftlichen Werterhaltung bzw. -veränderung verstehen sich die ,advocacy-groups“, zu denen z.B. Greenpeace und WWF gehören. Die Vielfalt der Erscheinungsformen wird durch folgende Abbildung verdeutlicht (über 200.000 Typen sind denkbar):

\begin{tabular}{|c|c|c|c|c|c|c|c|}
\hline Merkmale & \multicolumn{7}{|c|}{ Ausprägungen } \\
\hline $\begin{array}{c}\text { Primäre Nutz- } \\
\text { nießer }\end{array}$ & \multicolumn{2}{|c|}{ Mitglieder } & \multicolumn{3}{|c|}{ Dritte } & \multicolumn{2}{|c|}{ Allgemeinheit } \\
\hline Trägerschaft & \multicolumn{3}{|c|}{\begin{tabular}{r|r}
\multicolumn{2}{r}{ Staatlich } \\
\end{tabular}} & \multicolumn{4}{|c|}{ Privat } \\
\hline Rechtsform & $\begin{array}{l}\text { öffentlich- } \\
\text { rechtlich }\end{array}$ & \multicolumn{2}{|c|}{ Verein } & \multicolumn{3}{|c|}{ Genossenschaft } & Stiftung \\
\hline $\begin{array}{c}\text { Finanzierungs- } \\
\text { art }\end{array}$ & Preise & \multicolumn{2}{|c|}{$\begin{array}{l}\text { Mitglieder- } \\
\text { beiträge }\end{array}$} & & \multicolumn{2}{|c|}{$\begin{array}{l}\text { Subventio- } \\
\text { nen }\end{array}$} & $\begin{array}{l}\text { Staatsbei- } \\
\text { träge }\end{array}$ \\
\hline \multirow{2}{*}{ Leistungsart } & \multicolumn{3}{|c|}{ Materiell } & \multicolumn{4}{|c|}{ Immateriell } \\
\hline & \multicolumn{2}{|c|}{ Individualgüter } & \multicolumn{3}{|c|}{ Meritorische Güter } & \multicolumn{2}{|c|}{ öffentliche Güter } \\
\hline $\begin{array}{l}\text { Organisations- } \\
\text { teilnehmer }\end{array}$ & \multicolumn{2}{|c|}{ Profis, Funktionäre } & \multicolumn{3}{|c|}{ Gemischt } & \multicolumn{2}{|c|}{ Ehrenamtliche } \\
\hline $\begin{array}{l}\text { Organisations- } \\
\text { struktur }\end{array}$ & \multicolumn{3}{|c|}{ Hierarchisch } & \multicolumn{4}{|c|}{ Demokratisch } \\
\hline Lebensalter & \multicolumn{2}{|c|}{0 bis 49 Jahre } & \multicolumn{3}{|c|}{50 bis 99 Jahre } & \multicolumn{2}{|c|}{ über 100 Jahre } \\
\hline $\begin{array}{l}\text { Zahl der Träger/ } \\
\text { Mitglieder }\end{array}$ & \multicolumn{2}{|l|}{ bis 10} & bis 500 & \multicolumn{3}{|c|}{ bis 1000} & über 1000 \\
\hline Zahl der Profis & Keine & \multicolumn{2}{|c|}{1 bis 10} & \multicolumn{3}{|c|}{11 bis 50} & über 50 \\
\hline
\end{tabular}

\section{Abb. 1: Typologie von Nonprofit-Organisationen}

Quelle: $\quad$ In Anlehnung an Burla (1989), S. 78

Diese Organisationen bewegen sich in einem Kontinuum zwischen Markt und Staat. Zur Erfüllung ihrer Mission und zur langfristigen Sicherung der Existenz sind sie auf Ressourcen angewiesen. 


\section{Finanzielle Ressourcen}

Im Vergleich zu kommerziellen Unternehmen unterscheidet sich die Finanzierung von NPO in zwei wesentlichen Punkten. Wie Unternehmen können sie zum einen durch den Absatz marktfähiger Güter gegen Entgelt (z.B. Absatz von Versicherungen, Reisebüro des ADAC) Erlöse erzielen. Zum andern fließen ihnen neben den Leistungsentgelten finanzielle Mittel durch Mitgliederbeiträge, öffentliche Beiträge (Subventionen) und private Zuschüsse (Spenden) zu. Außerdem erhalten diese Organisationen nicht nur Finanzmittel, sondern zu einem wesentlichen Teil auch sog. Finanzmittelsurrogate (Zeit-, Sachspenden u.a).

Für die NPO stellen die Finanzmittel - neben anderen Mitteln - eine wichtige Ressourcenquelle dar. Diese Inputs werden einerseits aus dem Leistungsprozess generiert, so bspw. über Umsatzerlöse und Gebühren. Im Weiteren leistet auch die öffentliche Hand namhafte Beiträge in Form von leistungsbezogenen Subventionen zur Finanzierung des Dritten Sektors. Mithin stellt das Spendenwesen eine wichtige Einnahmequelle dar. Außerhalb des eigentlichen Leistungsprozesses besteht auch für NPO die Möglichkeit der Fremdfinanzierung durch Kredite. Dies ist allerdings durch die oftmals ungenügende Kreditwürdigkeit der NPO beschränkt. Auch haben NPO nicht die Möglichkeit, über die Ausgabe von Kapitalanteilen zusätzliche Finanzmittel zu beschaffen (Ausnahme bei Genossenschaften).

Die Existenz zahlreicher NPO wäre ohne das Vorhandensein von Finanzmittelsurrogaten gefährdet. Neben dem Geldmittelzufluss stellt die ehrenamtliche Arbeit in Führungsgremien sowie die Freiwilligenarbeit in ausführenden Tätigkeiten für viele NPO einen wesentlichen Input dar. Die unbezahlte Arbeit kann als Zeitspende - eine unverzichtbare Ressource - bezeichnet werden. Bei einzelnen Organisationen, vorwiegend in lokalen und regional tätigen NPO, spielen diese Surrogate eine bedeutsamere Rolle als die Finanzmittelzuflüsse. Neben Geld- und Zeitspenden sind auch Sachspenden und Spenden von Dienstleistungsanrechten zu erwähnen. Sachspenden können in Form von Immobilien (Grundstücke, Häuser) und Objekten (Kunstobjekte, Altkleider) auftreten. Sowohl bei Sach- als auch bei Zeitspenden stellt sich dabei die Frage der Bewertung. Während Geldspenden in der Schweiz in gewissen Grenzen steuerlich abzugsfähig sind, gilt dies nicht für Sach- und Zeitspenden. Im Fall der Sachspenden sind momentan gesetzgeberische Bestrebungen im Gange, diese steuerlich mit Geldspenden gleichzusetzen.

Neben den eigentlichen Finanzmitteln fallen unter die Kategorie der Finanzmittelsurrogate die sog. Dienstleistungsanrechte. Die Zur-Verfügung-Stellung von Räumen oder Fahrzeugen, Leihgaben von Kunstobjekten, von Werbezeiten an TV und Radio, von Inseratund Plakatierungsflächen sind Beispiele dafür. Dieser nicht finanzielle Zufluss sollte in Anwendung kalkulatorischer Grundsätze zumindest in Mengengrößen quantifiziert und in der Jahresrechnung von NPO ausgewiesen werden. Insbesondere im Fall der Zeitspenden können diese gegenüber der öffentlichen Hand auch als Legitimation öffentlicher Unterstützung dienen. Nach Möglichkeit sollte auch ein Wertansatz ermittelt werden. Trotz der damit verbundenen Bewertungsprobleme kann nur durch eine Bewertung der 
Finanzmittelsurrogate der Gesamtressourceneinsatz erfasst werden. Das Beispiel der Spenden lebender Materie verdeutlicht diese Problematik. Für einzelne Organisationen wie das Rote Kreuz stellen Blutspenden und weitere Spenden lebender Materie einen wichtigen Bestandteil ihrer Tätigkeit dar. In diesem Bereich erscheint die Bewertung besonders problematisch bzw. schwierig.

\section{Weitere Ressourcen}

Eine weitere noch schwerer zu bewertende Ressource stellen die Beiträge der Wissenslieferanten und das Nutzen von Netzwerken durch Mitglieder/Träger dar. Bereits die Gründung einer NPO bildet bereits ein Ergebnis erfolgreichen Wissensmanagements, nämlich eine Lernwerkstatt. Auf dem Markt wie gegenüber dem Staat suchten Betroffene durch die Gründung einer neuen Organisation zu kollektiv Beteiligten zu werden. Aus kleinen Zellen entstanden die Konsumgenossenschaften, die Gewerkschaften, die Wirtschaftsverbände sowie die Vereine aus dem Freizeitbereich. Das Einbringen von Wissen durch die Träger stärkt das Machtpotenzial der NPO ebenso wie die durch erfolgreiche Beziehungspflege erzielten Effekte. 


\section{Zusammenfassung}

In Anlehnung und Weiterentwicklung des von Gerhard Krönes entwickelten Analyserasters ergibt sich die folgende Struktur des Ressourcenportfolios:

\begin{tabular}{|c|c|}
\hline Art & $\begin{array}{l}\text { - Finanzmittel: } \\
\text { - } \quad \text { aus dem Leistungsprozess: Umsatzerlöse, Gebühren, } \\
\text { Beiträge, Spenden, Zuwendungen (leistungsbezogene } \\
\text { Subventionen) } \\
\text { - } \quad \text { außerhalb des Leistungsprozesses: Kredite, Kapital- } \\
\text { subventionen, (Finanz-)Legate } \\
\text { - } \quad \text { Finanzsurrogate: } \\
\text { - } \quad \text { Zeitspenden: ehrenamtliche bzw. Freiwilligen-Arbeit } \\
\text { für Führungs- und Ausführungsaufgaben, Dienstzutei- } \\
\text { lung von Arbeitskräften (,Lebendsubventionen“) } \\
\text { - } \quad \text { Sachspenden: Immobilien und Mobilien } \\
\text { - } \quad \text { Spenden von Dienstleistungsanrechten } \\
\text { - } \quad \text { Spenden lebender Materie (z.B. Blut, Organe) } \\
\text { - } \quad \text { Know-how, Beziehungsnetzwerke etc. }\end{array}$ \\
\hline Herkunft & $\begin{array}{l}\text { - intern: } \\
\text { Umsatzerlöse, Vermögenserträge, Vermögensum- } \\
\text { schichtungen, Mitgliederbeiträge, Einbringen von Wis- } \\
\text { sen und Beziehungen (net-working) } \\
\text { - extern: } \\
\text { Personen, private Haushalte, Unternehmen, private } \\
\text { NPO (allenfalls als potenzielle Mitglieder gewertet), } \\
\text { staatliche Körperschaften: Staat als Konsument, als } \\
\text { Hoheitsträger }\end{array}$ \\
\hline
\end{tabular}

Abb. 2: Ressourcen von Nonprofit-Organisationen: Eigenschaften und Ausprägungen

Quelle: Eigene Darstellung

Dank dieses Rasters ist es nicht nur möglich, den gesamten Input an Ressourcen (Finanzen und Finanzsurrogate) zu erfassen, zu bewerten und die relativen Anteile der Inputs festzustellen. Dieses Raster kann auch hilfreich sein für die Planung der Ressourcen und die Suche nach neuen Quellen der Ressourcen. 


\section{Literaturverzeichnis}

Anheier, Helmut K. (2003), Der Dritte Sektor in Zeiten des gesellschaftlichen Umbruchs, in: VerbandsManagement, 29. Jg., Heft 1, S. 38-47

Blümle, Ernst-Bernd und Reinbert Schauer (2002), Ansatz zur Analyse von Nonprofit-Organisationen, in: Der Schweizer Treuhänder, Schwerpunktnummer Rechnungslegung von Nonprofit Organisationen, Heft 6, S. 561566

Burla, Stephan (1989), Rationales Management in Nonprofit-Organisationen, Bern und Stuttgart

Krönes, Gerhard (2001), Finanzierung von Nonprofitorganisationen, Eine Betrachtung unter besonderer Berücksichtigung der Parteienfinanzierung, in: DBW, 61. Jg., S. 83 